\title{
UPPER BOUNDS FOR THE PERIMETER OF PLANE CONVEX BODIES
}

\author{
A. GLAZYRIN ${ }^{1, *}$ and F. MORIĆ ${ }^{2, \dagger}$ \\ ${ }^{1}$ Department of Mathematics, University of Texas at Brownsville, 80 Fort Brown, \\ Brownsville, TX 78520, USA \\ e-mail: alexey.glazyrin@utb.edu \\ ${ }^{2}$ Ecole Polytechnique Fédérale de Lausanne, SB MATHGEOM DCG Station 8, \\ CH-1015, Lausanne, Switzerland \\ e-mail: filip.moric@epfl.ch
}

(Received March 4, 2013; revised April 3, 2013; accepted April 5, 2013)

\begin{abstract}
We show that the maximum total perimeter of $k$ plane convex bodies with disjoint interiors lying inside a given convex body $C$ is equal to per $(C)+2(k-1)$ diam $(C)$, in the case when $C$ is a square or an arbitrary triangle. A weaker bound is obtained for general plane convex bodies. As a consequence, we establish a bound on the perimeter of a polygon with at most $k$ reflex angles lying inside a given plane convex body.
\end{abstract}

\section{Introduction}

It is well known that if $C$ and $D$ are two plane convex bodies such that $C \subseteq D$, then

$$
\operatorname{per}(C) \leqq \operatorname{per}(D)
$$

In other words, the maximum perimeter of a convex body lying inside a given convex body $D$ is equal to per $(D)$. While some generalizations of this classical result are known (in particular, the straightforward generalization to higher dimensions, see e.g. $[4,13,18]$ ), it comes as a surprise that the situation when several disjoint bodies lie inside another convex body, has not been investigated before. In this paper we are interested in answering the following general question:

* The authors gratefully acknowledge support from the Russian government project 11.G34.31.0053 and the Swiss National Science Foundation Grants 200020-144531 and 20021137574.

$\dagger$ Corresponding author.

Key words and phrases: convex body, diameter, perimeter, area, geometric inequality, simple polygon, reflex angle.

Mathematics Subject Classification: 52A10, 52A38, 52A40. 
QUESTION 1. For a given $k \in \mathbb{N}$, what is the maximum total perimeter of $k$ plane convex bodies with disjoint interiors lying inside a given plane convex body $D$ ?

Observe that for any $k$ and $D$ one can construct a family of convex bodies satisfying the conditions whose total perimeter is as close to per $(D)+2(k-1)$ diam $(D)$ as needed (see Fig. 1, left). Can we do better?
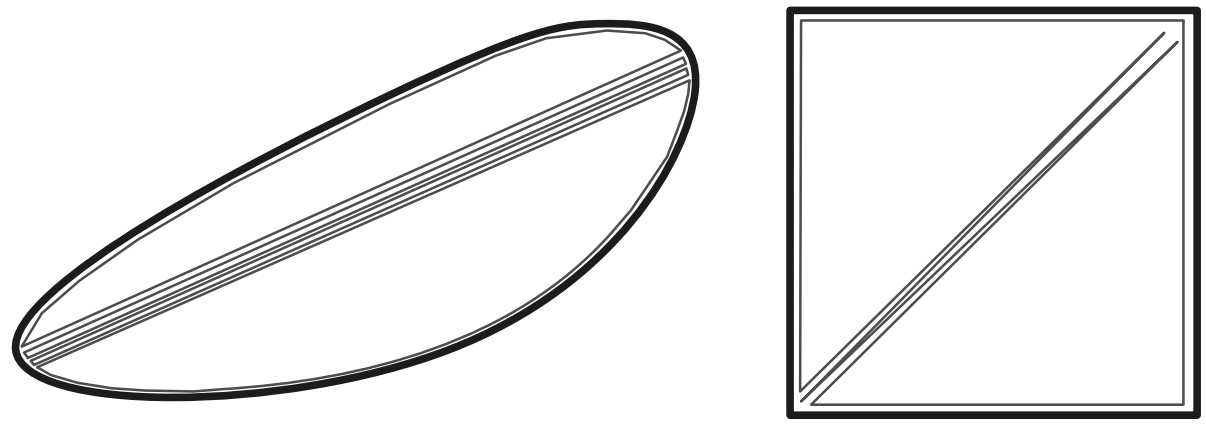

Fig. 1: Left: a family of $k$ convex bodies of total perimeter close to per $(S)+2(k-1)$ diam $(S)$. Right: a polygon with 2 reflex angles and (near) maximum perimeter inside a unit square

We show that if $D$ is a square, one cannot do better than per $(D)+$ $2(k-1) \operatorname{diam}(D)$.

TheOREM 1. If $C_{1}, \ldots, C_{k}$ are convex bodies with disjoint interiors lying inside a unit square, then

$$
\operatorname{per}\left(C_{1}\right)+\cdots+\operatorname{per}\left(C_{k}\right) \leqq 4+2 \sqrt{2}(k-1) .
$$

This inequality is tight.

Similarly, we have a tight result for the case of an arbitrary triangle.

TheOrem 2. Let $T$ be a triangle with longest side of length $d$. If $C_{1}$, $\ldots, C_{k}$ are convex bodies with disjoint interiors lying inside $T$, then

$$
\operatorname{per}\left(C_{1}\right)+\cdots+\operatorname{per}\left(C_{k}\right) \leqq \operatorname{per}(T)+2(k-1) d .
$$

This inequality is tight.

What happens for general figures? The following theorem, which is not tight, gives a good upper bound. 
THeOREm 3. If $C_{1}, \ldots, C_{k}$ are plane convex bodies with disjoint interiors lying inside a plane convex body $S$, then

$$
\sum_{i=1}^{k} \operatorname{per}\left(C_{i}\right) \leqq 1.22195 \operatorname{per}(S)+2(k-1) \operatorname{diam}(S) .
$$

We believe the constant 1.22195 in the last theorem can be reduced to 1 , giving a tight bound.

Conjecture 4. Given a plane convex body $S$ and $k \in \mathbb{N}$, the total perimeter of $k$ convex bodies with disjoint interiors lying inside $S$ is at most per $(S)+2 \operatorname{diam}(S)(k-1)$.

Let us note also that the proof of Theorem 3 gives the following result that does not need the condition that $S$ be convex.

TheOREM 5. If $C_{1}, \ldots, C_{k}$ are plane convex bodies with disjoint interiors lying inside a plane (not necessarily convex) body $S$, then

$$
\sum_{i=1}^{k} \operatorname{per}\left(C_{i}\right) \leqq \frac{4}{\sqrt{3}} \frac{\operatorname{area}(S)}{\operatorname{diam}(S)}+2 k \operatorname{diam}(S) .
$$

Note that the inequality of Theorem 5 is tight when $S$ is an equilateral triangle.

A generalization of our results to higher dimensions remains elusive. The following might be the right way to generalize.

Conjecture 6. Given a convex body $S \subset \mathbb{R}^{d}$ and $k \in \mathbb{N}$, the total surface area of $k$ convex bodies with disjoint interiors lying inside $S$ is at most $\operatorname{surf}(S)+2 \operatorname{sect}(S)(k-1)$, where $\operatorname{surf}(S)$ and sect $(S)$ denote respectively the surface area of $S$ and the maximum area of a section of $S$ by a hyperplane.

AuXiLIARY INEQUALities. The auxiliary results we used to prove Theorems 1 and 2 seem to be of independent interest. In fact, our main theorems are simple consequences of these inequalities. We show a refined version of the inequality per $(C) \leqq 4$ for a convex body $C$ inside a unit square.

Theorem 7. If $C$ is a convex body inside a unit square, then

$$
\operatorname{per}(C) \leqq 2 \sqrt{2}+(4-2 \sqrt{2}) \text { area }(C) .
$$

An analogous result that strengthens the well-known inequality per $(C)$ $\leqq \operatorname{per}(T)$ (see the notation below), is proven for a triangle.

THEOREM 8. Let $T$ be a triangle with longest side of length d. If $C$ is a convex body inside $T$, then

$$
\frac{\operatorname{per}(C)-2 d}{\operatorname{area}(C)} \leqq \frac{\operatorname{per}(T)-2 d}{\operatorname{area}(T)} .
$$


REMARK. At first, one might be tempted to believe that for any two convex bodies $C \subset D$ one has

$$
\frac{\operatorname{per}(C)-2 d}{\operatorname{area}(C)} \leqq \frac{\operatorname{per}(D)-2 d}{\operatorname{area}(D)},
$$

where $d=\operatorname{diam}(D)$. This is, however, false in general, already for quadrilaterals. Indeed, suppose that a polygon has a diameter that divides it into two polygons whose perimeters are $P_{1}+d$ and $P_{2}+d$, and whose areas are $A_{1}$ and $A_{2}$, respectively. Then it is easy to check that the inequality fails whenever

$$
\frac{P_{1}-d}{A_{1}} \neq \frac{P_{2}-d}{A_{2}}
$$

The above inequality is false even for some centrally symmetric polygons $D$ : e.g., if $D=a b c d e f$ is a regular hexagon and $C=$ ace.

Finally, in the proof of Theorem 3 we need the following inequality, that we prove in Section 3.

Theorem 9. For any plane convex body $C$ we have

$$
\frac{4}{\sqrt{3}} \frac{\operatorname{area}(C)}{\operatorname{diam}(C)}+2 \operatorname{diam}(C) \leqq 1.22195 \operatorname{per}(C) .
$$

Using standard inequalities (see Theorem 11 below), one can easily obtain a weaker inequality with the constant $1 / \sqrt{3}+1 \approx 1.577$ instead of 1.22195. The latter constant coincides in the first five digits with the tight constant. Although it is not needed for our purposes, it would be interesting to get a general form of Theorem 9 as described in the following question.

Question 2. For every $\alpha \in[0,1]$, determine the smallest constant $c(\alpha)$ such that for any plane convex body $C$ we have

$$
\alpha \text { area }(C)+(1-\alpha)(\operatorname{diam}(C))^{2} \leqq c(\alpha) \operatorname{per}(C) \operatorname{diam}(C) .
$$

The method we used to prove Theorem 9 can in principle give a tight constant for every fixed $\alpha$. It is not clear, however, how $c(\alpha)$ behaves as a function of $\alpha$. More generally, one may try to describe all inequalities of the form $\alpha$ area $(C)+\beta(\operatorname{diam}(C))^{2}+\gamma \operatorname{per}(C) \operatorname{diam}(C) \geqq 0$ that hold for every plane convex body $C$.

Relation to reflex angles. Let us present an application of our results to a problem that was our original motivation for this work. Consider a convex body $S$ of perimeter 1 . We know that any convex polygon contained in $S$ has perimeter at most 1 . However, as long as our polygon has at 
least one reflex angle (that is, an internal angle larger than $\pi$ ), its perimeter can exceed 1 (see Fig. 1, right). Intuitively, the more reflex angles our polygon has, the larger perimeter it can achieve. The following general question was posed by the second author at the 10th Gremo's Workshop on Open Problems [10]:

QUESTION 3. For a given $k$, what is the largest perimeter of a polygon having at most $k$ reflex angles that is contained in a given convex body of perimeter $p$ and diameter $d$ ?

Denote the number of reflex angles of a polygon $P$ by ref $(P)$. It is easy to see that any polygon $P$ can be cut into ref $(P)+1$ convex polygons. This allows us to use Theorems 1,2 and 3 to derive the following inequalities.

Theorem 10. If $P$ is a polygon contained in a plane convex body $S$, then

- $\operatorname{per}(P) \leqq \operatorname{per}(S)+2 \operatorname{ref}(P) \operatorname{diam}(S)$, if $S$ is a triangle or a square;

- $\operatorname{per}(P) \leqq 1.22195 \operatorname{per}(S)+2 \operatorname{ref}(P) \operatorname{diam}(S)$, in general.

Relation to total curvature. Interestingly, our results are also related to some classical inequalities bounding the perimeter of a curve inside a convex body in terms of its total curvature. An old result of Chakerian [5] says that for any curve $C$ in the plane (note that in this case we do not have a surrounding curve), the following inequality holds:

$$
L(C) \leqq \frac{1}{\sqrt{3}} \operatorname{diam}(C)(2+K(C)),
$$

where $L(C)$ and $K(C)$ are the length and the total absolute curvature of $C$, respectively (also, a generalization of this result to higher dimensions is given in [5]). Assuming that $C$ is a polygon whose internal angles are $\alpha_{1}, \ldots, \alpha_{n}$, we have

$$
K(C)=\sum_{i=1}^{n}\left|\pi-\alpha_{i}\right| \leqq 2 \pi+2 \pi \cdot \operatorname{ref}(C),
$$

which together with Chakerian's inequality gives an inequality similar to those in Theorem 10, but with worse constants.

The famous DNA theorem, conjectured by Tabachnikov [23] and proved by Lagarias and Richardson [15] and later in full generality by Nazarov and Petrov [19] (see also [20]), states that whenever a (possibly non-convex) closed curve $\gamma$ lies inside a convex closed curve $\Gamma$ (see Fig. 2), then $A K(\gamma)$ $\geqq A K(\Gamma)$, where by $A K$ we denote the average curvature, that is, the total absolute curvature of the curve divided by its perimeter. Similarly as above, with the notation from Theorem 10, this implies

$$
\operatorname{per}(P) \leqq \operatorname{per}(S)+\operatorname{ref}(P) \operatorname{per}(S)
$$




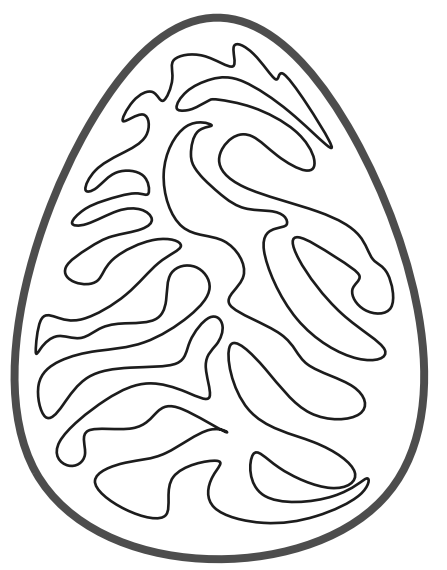

Fig. 2: DNA in a cell

which is again weaker than Theorem 10 .

Motivated by the above results, we can ask the following question.

QUESTION 4. For a given $k$, what is the largest perimeter of a polygon having total absolute curvature at most $k$ that is contained in a given convex body of perimeter $p$ and diameter $d$ ?

Related work. Geometric inequalities being a classical topic in discrete geometry, there is a vast related literature and numerous surveys, of which we recommend e.g. $[3,4,18]$. Bounding the perimeter of non-convex polygons was investigated by Audet, Hansen and Messine [1], who found for every $n$ a tight upper bound on the perimeter of a simple $n$-gon contained in a disk of radius one, thereby answering a question of Brass [3]. A different proof was given later by Dumitrescu [7], and also by Lángi [16] who gave some further extensions. In [8], Dumitrescu and Tóth solved the problem of finding (asymptotically) the maximum total perimeter of $n$ pairwise disjoint disks lying in the unit square $S$ and touching the boundary of $S$. Reinhard [21] determined the $n$-gons with a given diameter and maximal perimeter for all values of $n$ that are not powers of two, while the cases when $n=2^{r}$ for $r \geqq 3$ are still open [3]. Similarly, Bezdek and Fodor [2] proved the exact analogue of Reinhardt's theorem, with the same extremal configurations and with the same missing cases, for the maximum width in place of the maximum perimeter. Another old problem of Reinhardt [21] that asks for the maximum area of a convex $n$-gon of unit diameter is still open for even $n \geqq 10$ [3]. 


\section{Proofs of Theorems 1,2 and 3}

Theorem 1 is an easy consequence of Theorem 7 that is proven in the next section.

Proof of Theorem 1. Let $S$ be the unit square. By Theorem 7 we have

$$
\begin{array}{r}
\sum_{i=1}^{k} \operatorname{per}\left(C_{i}\right) \leqq 2 \sqrt{2} k+(4-2 \sqrt{2}) \sum_{i=1}^{k} \operatorname{area}\left(C_{i}\right) \\
\leqq 2 \sqrt{2} k+(4-2 \sqrt{2}) \text { area }(S)=4+2 \sqrt{2}(k-1) .
\end{array}
$$

In a similar manner, Theorem 2 follows from Theorem 8 .

Proof of Theorem 2. By Theorem 8 we have

$$
\sum_{i=1}^{k} \operatorname{per}\left(C_{i}\right) \leqq \frac{\operatorname{per}(T)-2 d}{\text { area }(T)} \sum_{i=1}^{k} \operatorname{area}\left(C_{i}\right)+2 d k \leqq \operatorname{per}(T)+2 d(k-1) \text {. }
$$

In the proof of Theorem 3 we will use some well-known inequalities for convex bodies, given in the next theorem.

TheOrem 11. For any plane convex body $C$ we have

(a) $[9,14]$ area $(C) \geqq \frac{\sqrt{3}}{4}(\operatorname{per}(C)-2 \operatorname{diam}(C)) \operatorname{diam}(C)$;

(b) $[13]$ per $(C) \leqq \pi \operatorname{diam}(C)$;

(c) [11] 4 area $(C) \leqq \operatorname{per}(C) \operatorname{diam}(C)$.

All the above inequalities are also listed in [22]. Note that in [12] the inequality (a) is quoted with a wrong constant.

Proof of TheOREM 3. We can assume without loss of generality that per $\left(C_{i}\right) \geqq 2 \operatorname{diam}(S)$, since we can discard those of the $k$ bodies for which that condition fails and apply induction to the rest (clearly, the inequality is true for $k=1$ ). By Theorem 11 we have

$$
\begin{gathered}
\sum_{i=1}^{k} \operatorname{area}\left(C_{i}\right) \geqq \frac{\sqrt{3}}{4} \sum_{i=1}^{k}\left(\operatorname{per}\left(C_{i}\right)-2 \operatorname{diam}\left(C_{i}\right)\right) \operatorname{diam}\left(C_{i}\right) \\
\geqq \frac{\sqrt{3}}{4} \sum_{i=1}^{k}\left(\operatorname{per}\left(C_{i}\right)-2 \operatorname{diam}(S)\right) \operatorname{diam}(S) .
\end{gathered}
$$

The last inequality is due to the fact that the function $f(x)=\left(\operatorname{per}\left(C_{i}\right)-\right.$ $2 x) x$ is decreasing for $x \geqq \frac{\operatorname{per}\left(C_{i}\right)}{4}$ and $\frac{\operatorname{per}\left(C_{i}\right)}{4} \leqq \operatorname{diam}\left(C_{i}\right) \leqq \operatorname{diam}(S)$ (note that we used Theorem 11). 
Rearranging terms and using the fact that $\sum$ area $\left(C_{i}\right) \leqq$ area $(S)$ and Theorem 9, we get

$$
\begin{aligned}
& \sum_{i=1}^{k} \operatorname{per}\left(C_{i}\right) \leqq \frac{4}{\sqrt{3}} \frac{\operatorname{area}(S)}{\operatorname{diam}(S)}+2 k \operatorname{diam}(S) \\
& \leqq 1.22195 \operatorname{per}(S)+2(k-1) \operatorname{diam}(S) .
\end{aligned}
$$

\section{Proof of Theorem 9}

In this section we give a proof of Theorem 9, based on an idea communicated to us by Dmitri Panov [6]. The proof reduces to the problem of computing the maximum of a certain trivariate function subject to several constraints, which we solve using the program Wolfram Mathematica 9.0. We use in the proof the notion of a circular segment which is a part of a disk cut off by a chord. The chord we call the base of the circular segment, and by the angle of the circular segment we mean its central angle in radians.

Proof of TheOrem 9. Without loss of generality we can assume that $\operatorname{diam}(C)=1$. By Theorem 11 we have that area $(C) \in\left[0, \frac{\pi}{4}\right]$. It suffices to prove the following statement: if $C$ is a plane convex body such that there are two points $a, b \in \partial C$ with $|a b|=1$ (this condition is equivalent with $\operatorname{diam}(C) \geqq 1)$, then

$$
\frac{\frac{4}{\sqrt{3}} \operatorname{area}(C)+2}{\operatorname{per}(C)} \leqq 1.22195 .
$$

To prove this inequality, let us consider two convex bodies $C_{1}$ and $C_{2}$ in which $C$ is split by the segment $a b$. The crucial observation is the following. For $i=1,2$, denote by $C_{i}^{\prime}$ the circular segment with base $a b$ such that area $\left(C_{i}^{\prime}\right)=$ area $\left(C_{i}\right)$ (we assume that $C_{i}^{\prime}$ lies on the same side of the line $a b$ as $\left.C_{i}\right)$. Then we claim that per $\left(C_{i}^{\prime}\right) \leqq \operatorname{per}\left(C_{i}\right)$. Indeed, if we denote by $C_{i}^{\prime \prime}$ the circular segment with base $a b$ lying on the opposite side of the line $a b$ from $C_{i}$ and such that $C_{i}^{\prime} \cup C_{i}^{\prime \prime}$ is a disk (Fig. 3), then using the isoperimetric inequality (see e.g. [4]) we have that

$$
\begin{aligned}
& \left(\operatorname{per}\left(C_{i}\right)+\operatorname{per}\left(C_{i}^{\prime \prime}\right)-2|a b|\right)^{2} \geqq 4 \pi\left(\operatorname{area}\left(C_{i}\right)+\operatorname{area}\left(C_{i}^{\prime \prime}\right)\right) \\
= & 4 \pi\left(\operatorname{area}\left(C_{i}^{\prime}\right)+\operatorname{area}\left(C_{i}^{\prime \prime}\right)\right)=\left(\operatorname{per}\left(C_{i}^{\prime}\right)+\operatorname{per}\left(C_{i}^{\prime \prime}\right)-2|a b|\right)^{2},
\end{aligned}
$$

which implies per $\left(C_{i}^{\prime}\right) \leqq \operatorname{per}\left(C_{i}\right)$, as needed. Hence, we have

$$
\frac{\frac{4}{\sqrt{3}} \operatorname{area}(C)+2}{\operatorname{per}(C)} \leqq \frac{\frac{4}{\sqrt{3}}\left(\operatorname{area}\left(C_{1}^{\prime}\right)+\operatorname{area}\left(C_{2}^{\prime}\right)\right)+2}{\operatorname{per}\left(C_{1}^{\prime}\right)+\operatorname{per}\left(C_{2}^{\prime}\right)-2|a b|} .
$$




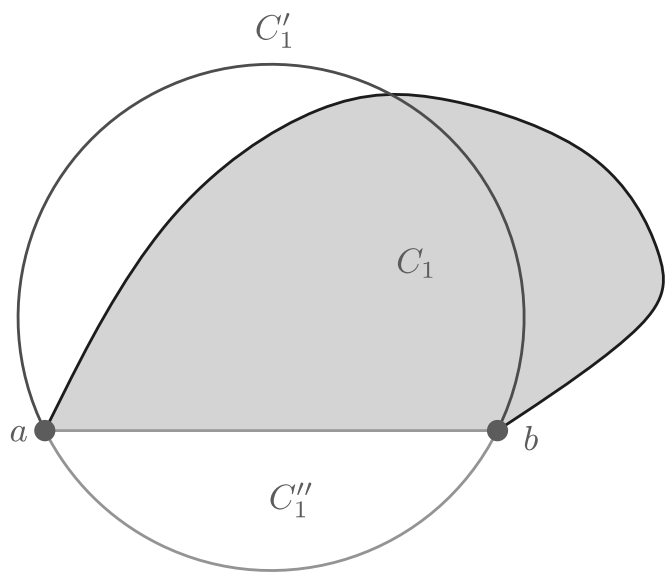

Fig. 3: Applying the isoperimetric inequality

Denote by $\theta_{1}$ and $\theta_{2}$ the angles of $C_{1}^{\prime}$ and $C_{2}^{\prime}$ (we have $\theta_{1}, \theta_{2} \in(0,2 \pi)$ ). It suffices to show that the maximum of the function

$$
f\left(A, \theta_{1}, \theta_{2}\right)=\frac{\frac{4}{\sqrt{3}} A+2}{\frac{\theta_{1}}{2 \sin \frac{\theta_{1}}{2}}+\frac{\theta_{2}}{2 \sin \frac{\theta_{2}}{2}}},
$$

subject to the constraints

$$
\frac{\theta_{1}-\sin \theta_{1}}{8 \sin ^{2} \frac{\theta_{1}}{2}}+\frac{\theta_{2}-\sin \theta_{2}}{8 \sin ^{2} \frac{\theta_{2}}{2}}=A, \quad 0 \leqq \theta_{1} \leqq 2 \pi, \quad 0 \leqq \theta_{2} \leqq 2 \pi, \quad 0 \leqq A \leqq \frac{\pi}{4},
$$

is not more than 1.22195. This we confirm, however, by using the program Mathematica. By executing the following commands

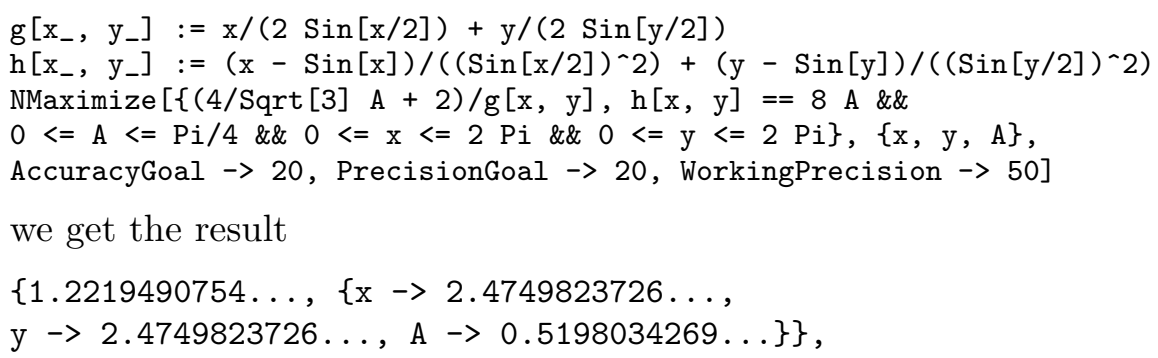

which shows that the maximum is equal to $1.2219490754 \ldots$, finishing the proof.

An idea for a simpler proof of Theorem 9 was given to us by Prof. Endre Makai [17]. It is shown in [13], Aufgabe 77a on page 53, that among all convex figures of diameter $d$ and perimeter $p$, the largest area is attained by 
the union of two identical circular segments whose common base is a segment of length $d$ (and the segments lie on the opposite sides of the base). Using this result, in order to prove Theorem 9, it suffices to find

$$
\max _{0 \leqq \theta \leqq \pi} \frac{\frac{1}{\sqrt{3} \sin ^{2}(\theta / 2)}(\theta-\sin \theta)+2}{\frac{\theta}{\sin (\theta / 2)}} .
$$

Again with the help of Mathematica, we get that the maximum is equal to $1.2219490754 \ldots$ (attained at $\theta=2.4749823726 \ldots$ ), which coincides with the result we obtained before.

Another way to simplify the above proof of Theorem 9 , proposed by the referee, is to perform first a Steiner symmetrization on $C$ in direction $a b$. In this way, the diameter and the area remain the same, while the perimeter does not increase. Also, the resulting figure will be symmetric with respect to $a b$, so we can continue the analysis as before, with the assumption that area $\left(C_{1}\right)=$ area $\left(C_{2}\right)$, which would in turn simplify the constrained maximization problem we face at the end.

REMARK. One can see from the proof that Theorem 9 is "tight" in the sense that the first five significant digits of the constant on the right hand side cannot be improved.

\section{Proofs of Theorems 7 and 8}

We start with a few standard lemmas that we need for the proof.

LEMma 4.1. The function $f(x)=\sqrt{(x-a)^{2}+b^{2}}+\sqrt{(x+a)^{2}+b^{2}}$ is strictly decreasing for $x \leqq 0$ and strictly increasing for $x \geqq 0$.

Proof. Since the function is even, it suffices to show that it is increasing for $x \geqq 0$. We will show that $f^{\prime}(x)>0$ for $x>0$. We calculate

$$
f^{\prime}(x)=\frac{x-a}{\sqrt{(x-a)^{2}+b^{2}}}+\frac{x+a}{\sqrt{(x+a)^{2}+b^{2}}},
$$

so the inequality $f^{\prime}(x)>0$ is obvious if $x>a$ or $a=0$, while for $0<x \leqq a$ it is equivalent to

$$
(a-x) \sqrt{(x+a)^{2}+b^{2}}<(x+a) \sqrt{(x-a)^{2}+b^{2}},
$$

which is, after squaring, equivalent to $b^{2} a x>0$. 
Corollary 4.1. If abcd is a trapezoid such that $a b \| c d$ and $\angle b a d>$ $\angle a b c$, then

$$
|a d|+|b d|>|a c|+|b c| .
$$

Proof. Let $d^{\prime}$ be the symmetric image of $d$ with respect to the perpendicular bisector of $a b$. Then $c$ lies on the segment $d d^{\prime}$. Without loss of generality, suppose that $|c d|>\left|c d^{\prime}\right|$. Lemma 4.1 implies that

$$
|a c|+|b c|<\left|a d^{\prime}\right|+\left|b d^{\prime}\right|=|a d|+|b d| .
$$

Lemma 4.2. Let $S$ be a plane convex body. If $C$ is a convex polygon lying inside $S$, then there exists a polygon $C^{\prime}$ whose all vertices lie on the boundary of $S$ and such that area $(C)=$ area $\left(C^{\prime}\right)$ and $\operatorname{per}(C) \leqq \operatorname{per}\left(C^{\prime}\right)$.

Proof. Among the convex bodies $C^{\prime} \subseteq S$ such that area $(C)=$ area $\left(C^{\prime}\right)$, choose one for which per $\left(C^{\prime}\right)$ is maximum. Denote by $n$ the number of vertices of $C^{\prime}$ and let $C^{\prime}=a_{1} a_{2} \ldots a_{n}$. We claim that $C^{\prime}$ has no internal vertices, i.e., all its vertices lie on the boundary of $S$ as needed. Suppose the contrary. Let $a_{i}$ be an internal vertex. By Lemma 4.1 we can move the vertex $a_{i}$ along a vector parallel to $a_{i-1} a_{i+1}$, so that the area of $C^{\prime}$ remains the same, while its perimeter strictly increases. This would contradict the choice of $C^{\prime}$.

Proof of Theorem 7 . Let $S=a b c d$ be the given unit square. If suffices to prove the statement for the case when $C$ is a convex polygon. Moreover, by Lemma 4.2 we can assume that the vertices of $C$ lie on the boundary of the square. The goal is to show that the function

$$
f(C)=\operatorname{per}(C)-(4-2 \sqrt{2}) \text { area }(C)
$$

attains its maximum for $C=S$, or, in other words, we need to show that for any convex polygon $C$ whose vertices are on the boundary of $S$ we have $f(C) \leqq f(S)=2 \sqrt{2}$.

Suppose that $C$ has two consecutive vertices lying on two consecutive sides of the square, say, $x$ lying on $a b$ and $y$ lying on $b c$, while the triangle $x b y$ is disjoint from $C$ (see Fig. 4, left). Let $C^{\prime}$ be the union of $C$ and the triangle $x b y$. Denote $|b x|=p$ and $|b y|=q$ and assume that $p, q>0$. The inequality $f(C) \leqq f\left(C^{\prime}\right)$ is equivalent to

$$
\begin{gathered}
\sqrt{p^{2}+q^{2}} \leqq p+q-(2-\sqrt{2}) p q \Leftrightarrow \\
0 \leqq(\sqrt{2}-1) p q-\sqrt{2}(p+q)+\sqrt{2}+1 .
\end{gathered}
$$

The last inequality is easy to verify: the function $\varphi(p, q)=(\sqrt{2}-1) p q$ $-\sqrt{2}(p+q)+\sqrt{2}+1$ is linear in $q$, while $\varphi(p, 0)>0$ and $\varphi(p, 1) \geqq 0$. We 

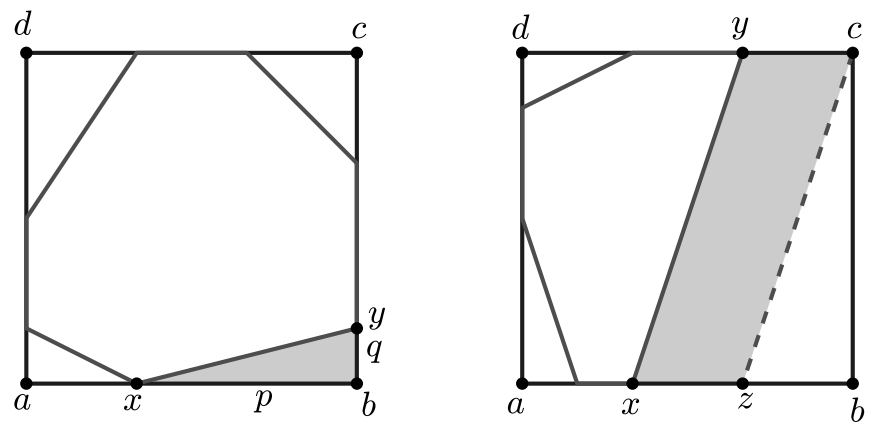

Fig. 4: Gluing the yellow part increases $f(C)$

showed that in this case $f(C) \leqq f\left(C^{\prime}\right)$, that is, by gluing the corner triangle $x b y$ to $C$ the value of $f(C)$ does not decrease. Let us call this a 1-operation.

Suppose now that $C$ has two consecutive vertices on two opposite sides of the square, e.g., $x$ on $a b$ and $y$ on $c d$, such that the quadrilateral $x b c y$ is disjoint from $C$ (see Fig. 4, right). Assume without loss of generality that $|b x| \geqq|c y|$ and let $z$ be a point on the segment $x b$ such that $|x z|=|c y|$. Denote by $C^{\prime}$ the union of $C$ and the parallelogram $x z c y$ and let $C^{\prime \prime}$ be the union of $C$ and the quadrilateral $x b c y$. We have $f(C) \leqq f\left(C^{\prime}\right) \leqq f\left(C^{\prime \prime}\right)$, where the first inequality is obvious, while the second one follows from what we proved before. We showed that in this case by gluing the quadrilateral $x b c y$ to $C$ the value $f(C)$ does not decrease. We call this a 2-operation.

Finally, suppose that $C$ is a "corner triangle", e.g., $C=a x y$, where $x$ and $y$ are points on the sides $a b$ and $a d$, respectively. Denoting $|a x|=p$ and $|a y|=q$, the inequality $f(C) \leqq 2 \sqrt{2}$ is equivalent to

$$
p+q+\sqrt{p^{2}+q^{2}} \leqq 2 \sqrt{2}+(2-\sqrt{2}) p q .
$$

Since $\sqrt{p^{2}+q^{2}} \leqq \sqrt{2} \max \{p, q\}$, assuming $p \geqq q$, it suffices to show the inequality

$$
(\sqrt{2}+1) p+q \leqq 2 \sqrt{2}+(2-\sqrt{2}) p q,
$$

which is easy to check (due to linearity, it is enough to check the cases $q=0$ and $q=1$ ).

It remains to notice that by applying (possibly several) 1-operations and 2-operations to any polygon $C$ whose vertices lie on the boundary of $S$, we can obtain either a corner triangle or the whole square. This finishes the proof.

The strategy in proving Theorem 8 is similar, although the proof is slightly more difficult. We need an additional lemma. 
Lemma 4.3 (Ptolemy Inequality). If $a, b, c, d$ are points in the plane, then

$$
|a b| \times|c d|+|b c| \times|a d| \geqq|a c| \times|b d| .
$$

Proof of Theorem 8. Let $T=a b c$ be the given triangle. As in the proof of Theorem 7, by Lemma 4.2 we may assume that $C$ is a polygon whose all vertices lie on the boundary of $T$. For technical reasons, it is convenient to consider interchangeably the following two functions:

$$
f(C)=\frac{\operatorname{per}(C)-2 d}{\operatorname{area}(C)} \quad \text { and } \quad g(C)=\operatorname{per}(C)-\frac{\operatorname{per}(T)-2 d}{\operatorname{area}(T)} \cdot \operatorname{area}(C),
$$

so that the inequality that we need to prove becomes equivalent to $f(C)$ $\leqq f(T)$ and also to $g(C) \leqq 2 d$. We first prove the inequality in two special cases.

Suppose that $C$ is a triangle that shares a side with $T$, say, $C=a b x$, where $x$ lies on the side $b c$. Let us use the following notation: $|a b|=p$, $|b x|=q,|c x|=s,|a c|=t$. The inequality $f(C) \leqq f(T)$ is equivalent to

$$
\frac{p+q+r-2 d}{\frac{1}{2} p q \sin \angle a b c} \leqq \frac{p+q+s+t-2 d}{\frac{1}{2} p(q+s) \sin \angle a b c} \Leftrightarrow p s+q r+r s \leqq 2 d s+q t .
$$

From Theorem 4.3 we have that

$$
p s+r(q+s) \leqq 2 p s+q t \leqq 2 d s+q t,
$$

so in this case we are done.

As for the next case, suppose that $C$ is a "corner" triangle, e.g., $C=a x y$, where $x$ and $y$ are on the sides $a b$ and $a c$, respectively. Since either $\angle a b c \geqq$ $\angle a x y$ or $\angle a c b \geqq \angle a y x$, we can assume without loss of generality that $\angle a c b \geqq$ $\angle a y x$. Let $z$ be the point on $a b$ such that $x y \| c z$ (see Fig. 5, left). Denoting $\frac{|a c|}{|a y|}=\frac{|a z|}{|a x|}=k \geqq 1$ and $C^{\prime}=a c z$, we have that the inequality $f(C) \leqq f\left(C^{\prime}\right)$ is equivalent to

$$
\frac{\operatorname{per}(C)-2 d}{\text { area }(C)} \leqq \frac{k \operatorname{per}(C)-2 d}{k^{2} \text { area }(C)} \Leftrightarrow k \operatorname{per}(C) \leqq 2 d(k+1) .
$$

The last inequality is true, since

$$
k \operatorname{per}(C)=\operatorname{per}\left(C^{\prime}\right) \leqq 3 d \leqq 2 d(k+1) .
$$

By the preceding case $f\left(C^{\prime}\right) \leqq f(T)$, so we can conclude that $f(C) \leqq f(T)$.

Having covered two special cases, we continue with the general case. By compactness, the function $g(C)$ attains its maximum for some convex 

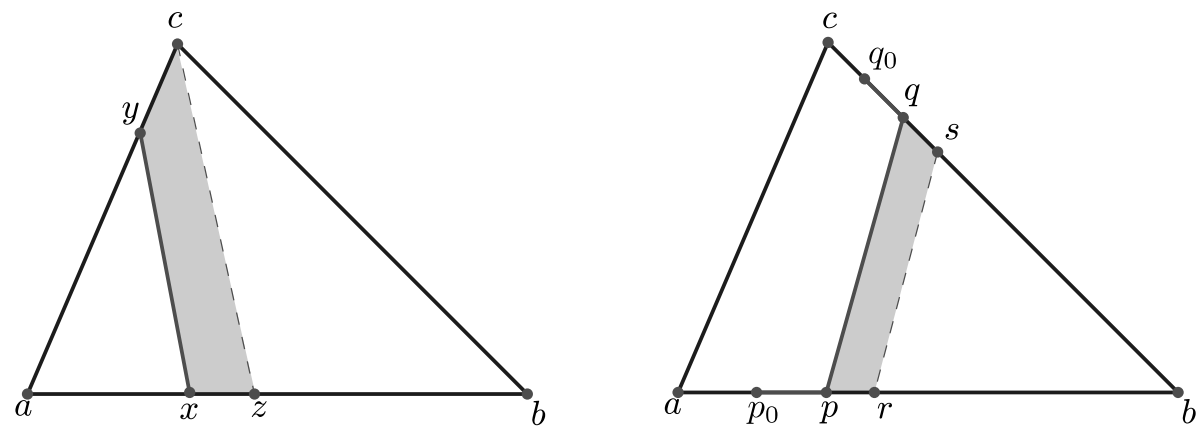

Fig. 5: Left: gluing the yellow part increases $f(C)$. Right: sliding the edge pq in one or the other direction increases $g(C)$

polygon $C$ (indeed, the set of convex polygons whose all vertices lie on the boundary of $T$, including the degenerate ones as well, can be considered as compact, and the function $g$ is certainly continuous and well-defined on this set). From now on, we suppose that $C$ is a polygon for which $g(C)$ is maximum.

We claim that it cannot happen that two non-consecutive sides of $C$ lie on two different sides of $T$. Suppose the contrary, e.g., let $p_{0}, p, q_{0}, q$ be distinct vertices of $C$ such that $p_{0}, p \in a b$ and $q_{0}, q \in b c$. Moreover, suppose that $p \in p_{0} b$ and $q \in q_{0} b$. Denote $|p b|=m,|q b|=n$ and $|p q|=\ell$. We can assume without loss of generality that the distance of $a$ from the line $p q$ is not larger than the distance of $c$ from the line $p q$. For all $x \in[0, m)$, let $C(x)$ be the union of $C$ and the trapezoid prsq, where $r \in p b, s \in q b, r s \| p q$ and $|p r|=x$ (see Fig. 5, right). Similarly, for all $x \in(m-|a b|, 0]$ let $C(x)$ be the (set-theoretic) difference of $C$ and the trapezoid prsq, where $r \in p a$, $s \in q c, r s \| p q$ and $|p r|=-x$. According to our assumption, the function $\varphi(x)=g\left(C_{x}\right)$ should attain its local maximum for $x=0$. We will show that this cannot be the case. Denote per $(C)=P$, area $(C)=A$ and let $h$ be the distance from $b$ to the line $p q$. It is easy to see that

$$
\begin{gathered}
\varphi(x)=P+x+\left(\ell-\frac{\ell}{m} x\right)+\frac{n}{m} x-\ell-\left(A+\left(\ell-\frac{\ell}{2 m} x\right) \frac{h}{m} x\right) \cdot \tau \\
=P-A \tau+\frac{m-\ell+n-\ell h \tau}{m} \cdot x+\frac{\ell h \tau}{2 m^{2}} \cdot x^{2}
\end{gathered}
$$

where

$$
\tau=\frac{\operatorname{per}(T)-2 d}{\operatorname{area}(T)}
$$

Since $\varphi(x)$ is a quadratic function in $x$ with a positive coefficient of the second degree term, it has no local maxima, which contradicts our assumption. 

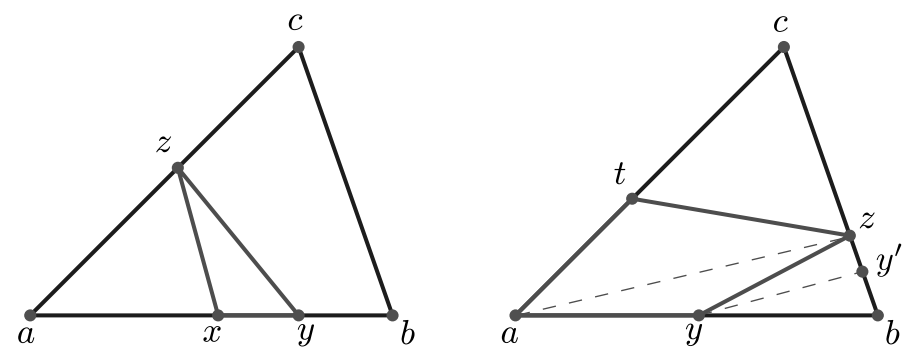

Fig. 6: Left: Case 1; Right: Case 2 when $x=a$

Now one can easily check that $C$ has to be of one of the forms covered by the following two cases.

Case 1: $C$ is a triangle with two vertices on one side of $T$ (and the third vertex on another side). Without loss of generality, let $C=x y z$, where $x, y \in a b$ and $z \in a c$, so that $x$ is between $a$ and $y$ (Fig. 6, left). Denote by $C^{\prime}$ the triangle ayz. Recall that we have proven the theorem for the case of a corner triangle. Now we can apply that fact two times. Let $d^{\prime}$ be the diameter of $C^{\prime}$. Since $C$ is a corner triangle for $C^{\prime}$ we have

$$
\frac{\operatorname{per}(C)-2 d^{\prime}}{\operatorname{area}(C)} \leqq \frac{\operatorname{per}\left(C^{\prime}\right)-2 d^{\prime}}{\operatorname{area}\left(C^{\prime}\right)} .
$$

Similarly, $C^{\prime}$ is a corner triangle for $T$ and we have

$$
\frac{\operatorname{per}\left(C^{\prime}\right)-2 d}{\operatorname{area}\left(C^{\prime}\right)} \leqq \frac{\operatorname{per}(T)-2 d}{\operatorname{area}(T)} .
$$

Adding the two inequalities we get

$$
\begin{gathered}
\frac{\operatorname{per}(C)-2 d}{\operatorname{area}(C)} \leqq \frac{\operatorname{per}(T)-2 d}{\operatorname{area}(T)}-2\left(d-d^{\prime}\right)\left(\frac{1}{\operatorname{area}(C)}-\frac{1}{\text { area }\left(C^{\prime}\right)}\right) \\
\leqq \frac{\operatorname{per}(T)-2 d}{\operatorname{area}(T)},
\end{gathered}
$$

where we used that area $\left(C^{\prime}\right) \geqq$ area $(C)$ and $d \geqq d^{\prime}$.

Case 2: $C$ is a quadrilateral with two vertices on one side of $T$ and one vertex on each of the other two sides. Without loss of generality, suppose that $C=x y z t$, where $x, y \in a b, z \in b c, t \in c a$ and $x \in a y$ (we allow the possibility that $x=y$ or $x=a$ ).

First we address the easier case when $x=a$, that is, $X=a y z t$ (Fig. 6 , right). Since

$$
\angle b a z+\angle c a z<\angle b z a+\angle c z a,
$$


we have that either $\angle b a z<\angle b z a$ or $\angle c a z>\angle c z a$. Without loss of generality suppose that $\angle b a z<\angle b z a$. Let $y^{\prime}$ be the point on segment $b z$ such that $y y^{\prime} \| a z$. Denoting $C^{\prime}=a y^{\prime} z t$ and applying Corollary 4.1 to the trapezoid $a z y^{\prime} y$, we have that $g\left(C^{\prime}\right)>g(C)$ (since area $(C)=$ area $\left(C^{\prime}\right)$ and per $\left(C^{\prime}\right)$ $>$ per $(C)$ ), which contradicts the choice of $C$.
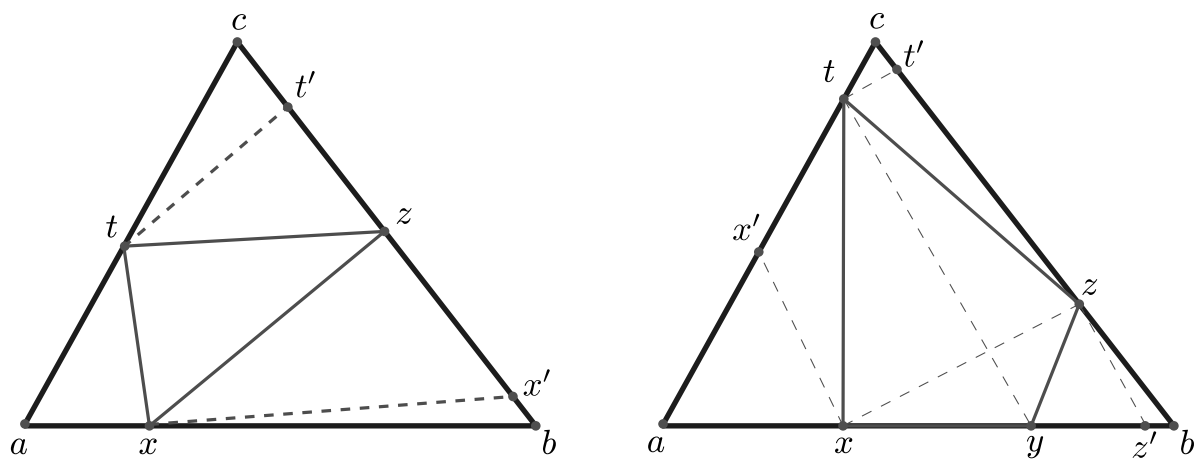

Fig. 7: Left: Case 2 when $x=y$. Right: Case 2, general situation

Next we consider the case when $x=y$, that is, $C$ degenerates into a triangle (Fig. 7, left). Without loss of generality, suppose that $x z$ is the longest side of the triangle $C=x z t$. Clearly, $\angle b x t+\angle x t z>\pi$, since otherwise we could move the point $x$ in the direction parallel to $z t$, so that the area of $x z t$ remains the same, while the perimeter strictly increases. Thus, we can pick a point $x^{\prime} \in b z$ such that $x x^{\prime} \| z t$. By Corollary 4.1 we have $\angle x t z \geqq \angle b z t$ (otherwise, we would have $g\left(x^{\prime} z t\right)>g(C)$ ). The line through $t$ parallel to $x z$ intersects either the segment $a x$ or the segment $c z$. Without loss of generality, we assume it intersects $c z$ at a point $t^{\prime}$. We have

$$
\begin{gathered}
\angle c z x=\angle c z t+\angle t z x=\pi-\angle b z t+\angle t z x \\
\geqq \pi-\angle x t z+\angle t z x=\angle t x z+2 \angle t z x>\angle t x z,
\end{gathered}
$$

which by Corollary 4.1, applied to the trapezoid $x z t^{\prime} t$, gives $g\left(x z t^{\prime}\right)>g(C)$. Contradiction.

In the rest of the proof we assume that $x \neq a$ and $x \neq y$ (see Fig. 7, right). Note that the inequality

$$
\angle a t y+\angle c t y+\angle b z x+\angle c z x>\angle a y t+\angle z y t+\angle t x z+\angle b x z
$$

holds since the left hand side is equal to $2 \pi$ and the right hand side is the sum of two angles of the quadrilateral xyzt. Thus, one of the following four cases has to happen: either $\angle a t y>\angle a y t$, or $\angle c t y>\angle z y t$, or $\angle b z x>\angle b x z$, or $\angle c z x>\angle t x z$. 
Suppose $\angle a t y>\angle a y t$. Let $x^{\prime}$ be the point the segment at such that $x x^{\prime} \| t y$. Denote $C^{\prime}=x^{\prime} y z t$. By Corollary 4.1, it follows that $g\left(C^{\prime}\right)>g(C)$, which is a contradiction.

Similarly, we get a contradiction assuming that $\angle b z x>\angle b x z$. Therefore, we must have $\angle b z x \leqq \angle b x z$.

Assume now that $\angle c t y>\angle z y t$. If the line through $z$ parallel to ty intersects the segment $c t$, we have a contradiction by Corollary 4.1 as before. Hence we suppose that this line intersects the segment by. If $\angle z t y<\angle b y t$, we again have a contradiction by Corollary 4.1 , so we can assume that $\angle z t y$ $\geqq \angle$ byt. Now we have two cases, depending on whether the line through $t$ parallel to $x z$ intersects the segment $a x$ or the segment $c z$. First, let us consider the case when it intersects the segment $a x$. Then we have that $\angle t z x \geqq \angle a x z$. It follows that

$$
\pi>\angle z t y+\angle t z x \geqq \angle b y t+\angle a x z=2 \pi-\angle b x z-\angle a y t>\pi,
$$

which is a contradiction. It remains to consider the case when the line through $t$ parallel to $x z$ intersects the segment $c z$. Similarly as before, we must have $\angle t x z \geqq \angle c z x$. This implies

$$
\angle b x t=\angle b x z+\angle t x z \geqq \angle b z x+\angle c z x=\pi,
$$

with a clear contradiction.

Analogously, the assumption $\angle c z x>\angle t x z$ also leads to a contradiction.

Acknowledgements. We are grateful to János Pach and Endre Makai for their helpful advice and to Emo Welzl for organizing the GWOP workshop, where this work was initiated. We would also like to thank Isaac Mabillard for his help in dealing with the program Mathematica. Finally, we are indebted to the anonymous referee for useful feedback.

\section{References}

[1] C. Audet, P. Hansen and F. Messine, Simple polygons of maximum parameter contained in the unit disk, Discr. Comput. Geom., 41 (2009), 208-215.

[2] A. Bezdek and F. Fodor, On convex polygons of maximal width, Arch. Math., 74 (2000), 75-80.

[3] P. Brass, W. Moser and J. Pach, Research Problems in Discrete Geometry, Springer (New York, 2005).

[4] Yu. D. Burago and V. A. Zalgaller, Geometric Inequalities, Springer-Verlag (Berlin, 1988).

[5] G. D. Chakerian, On some geometric inequalities, Proc. Amer. Math. Soc., 15 (1964), 886-888.

[6] D. Panov, Answer to question, http://mathoverflow.net/questions/120676/anotherdiameter-perimeter-area-inequality. 
[7] A. Dumitrescu, Metric inequalities for polygons, manuscript (June 2012).

[8] A. Dumitrescu and C. D. Tóth, Packing disks that touch the boundary of a square, in: 2nd Annual Fall Workshop on Computational Geometry, University of Maryland (2012).

[9] J. Favard, Problème d'extremums relatifs aux courbes convexes (premier mémoire), Ann. scient. de l'E. N. S. $3^{e}$ série, 46 (1929), 345-369.

[10] 10th GWOP Problem Booklet (internally distributed), ETH (Zürich, 2012), http:// www.ti.inf.ethz.ch/ew/workshops/gwop12.

[11] T. Hayashi, The extremal chords of an oval, Tôhoku Math. J., 22 (1923), 387-393.

[12] M. Henk and G. A. Tsintsifas, Some inequalities for planar convex figures, Elem. Math., 49 (1994), 120-124.

[13] I. M. Jaglom and W. G. Boltjanski, Konvexe Figuren, Hochschulbücher für Math. 24, VEB Deutscher Verl. Wiss (Berlin, 1956).

[14] T. Kubota, Eine Ungleichheit für Eilinien, Math. Z., 20 (1924), 264-266.

[15] J. C. Lagarias and T. J. Richardson, Convexity and the average curvature of plane curves, Geometriae Dedicata, 67 (1997), 1-30.

[16] Z. Lángi, On the perimeters of simple polygons contained in a disk, Monatsh. Math., 162 (2011), 61-67.

[17] E. Makai, Personal communication.

[18] D. S. Mitrinović, J. E. Pečarić and V. Volenec, Recent Advances in Geometric Inequalities, Kluwer Academic Publishers (London, 1989).

[19] A. I. Nazarov and F. V. Petrov, On Tabachnikov's conjecture, St. Petersburg Math. J., 19 (2008), 125-135.

[20] I. Pak, Lectures on Discrete and Polyhedral Geometry, preprint.

[21] K. Reinhardt, Extremale Polygone gegebenen Durchmessers, Jahresbericht Deutsch. Math.-Ver., 31 (1922), 251-270.

[22] P. R. Scott and P. Wah, Awyong, Inequalities for convex sets, Journ. of Ineq. in Pure and Appl. Math., 1(1) (2000), Article 6.

[23] S. Tabachnikov, The tale of a geometric inequality, in: MASS Selecta, AMS (Providence, RI, 2003), pp. 257-262. 